\title{
Impact of Urbanization on the Value of Ecosystem Services in Nanping City, China
}

\author{
Lili Zhao", ${ }^{1,2}$ Xuncheng Fan ${ }^{1,2 *}$, Han Lin², Tao Hong², Wei Hong² \\ ${ }^{1}$ College of Urban and Rural Construction, Shaoyang University, Shaoyang, 422000 China \\ ${ }^{2}$ Forestry College, Fujian Agriculture and Forestry University, 350002 Fuzhou, China
}

Received: 23 April 2020

Accepted: 18 June 2020

\begin{abstract}
The optimization of regional land use structures and functions and the sustainable use of the ecological environment are very important in the process of urbanization. In this study, based on the Landsat 7+ETM images of Nanping City from 2015, the object-oriented land use classification method was used to calculate the value of ecosystem services in Nanping City and various counties (districts).The spatial distribution characteristics between the levels of population urbanization, spatial urbanization, economic urbanization, and living urbanization and the value of ecosystem services were quantitatively analyzed by utilizing spatial measurement methods. The impact of the levels of four types of urbanization on the spatial distribution of ecosystem services values was explored using the bivariate spatial autocorrelation method. The results showed that there was a significant negative spatial correlation between population urbanization and the total value of ecosystem services. Specifically, the negative correlation between population urbanization and food production services was the most significant. Additionally, there was a certain positive correlation between economic urbanization and waste disposal services. Further, the bivariate LISA figures between different urbanization levels and the values of ecosystem services showed that a high urbanization-low ecosystem services value (High-Low) appeared in Jian'ou and Shaowu, while the regions of Guangze and Shunchang reflected a low urbanization-high ecosystem services value (Low-High).The findings of this study can provide information for decision-making regarding the sensible use of regional land resources and the improvement of ecological and environmental quality.
\end{abstract}

Keywords: land use, bivariate spatial autocorrelation, Fujian, non-agricultural industries

\section{Introduction}

Urbanization is a historical process that has gradually transformed traditional rural societies to modern urban

*e-mail: xunchengfan@126.com societies and has occurred with the development of social productivity, advances in technology, and the readjustment of the industrial structure of a country or region [1-2]. Urbanization is the movement of human society towards economic development and is of great significance to the improvement of the quality of life. Urbanization is an important indicator of the levels of economic, societal, cultural, 
and technological development in a country or region [3-5]. Furthermore, the urbanization process has an extensive and profound influence on the structure, function, and processes of ecosystems [2].

Ecosystem services are all the benefits that humans gain from ecosystems, including supply services (such as providing food and water), regulating services (such as controlling floods and diseases), cultural services (such as spiritual, recreational, and cultural benefits), and support services (such as maintaining a nutrient cycle in the living environment of the earth) [6]. The resources needed for human survival and development are ultimately derived from natural ecosystems. They not only provide food, medicine, and other raw materials for production, but also create and maintain the life support system of the earth and form the environmental conditions necessary for human survival. They also offer opportunities and spaces for leisure activities and entertainment, provide aesthetic resources, and supply the material basis for sustainable development [7-8].

The mechanism of interaction between urbanization and ecosystem services is complex. On the one hand, cities optimize the ecological environment and improve ecosystem services by exerting scale effects, optimizing industrial structure, and improving the quality of life of the urban population as well as the level of urban management [9]. A good ecosystem can provide the necessary materials and resources such as land, water, and fresh air to enhance a city's ecological competitiveness and improve the quality of life, thus supporting the sustainable development of a city [10-11]. On the other hand, changes in land use types caused by human activities often cause ecosystem degradation in the process of urbanization [12-14]. Liu et al. [15] found that urban expansion, manufacturing, mining and ports, and other human activities had a profound impact on the ecosystem value of the Bohai Rim coastal area. González-garcía et al. [16] found that urban expansion led to an imbalance between the supply and demand of ecosystem services in the Madrid region (Spain). Dewan and Yamaguchi et al. [17] discovered that urban expansion caused by elevation, population growth, and economic growth led to a significant reduction in the area of water bodies, cultivated land, vegetation, and wetlands in the Dhaka area. Therefore, studying the spatial correlation characteristics of urbanization and the value of ecological services can not only optimize the regional land use structure and functions in the process of rapid urbanization, but can also provide a scientific basis for ecosystem conservation and the maintenance of ecological security.

Nanping City is located in the southeast of China, $330 \mathrm{~km}$ from Taiwan Island (linear distance). Nanping City, including the surrounding areas, is based in Fujian Province and is a regional economic complex located in the core of the economic development zone on the west side of the strait. It is involved in working with Taiwan, unifying China, and furthering the Chinese economy on a global scale. Nanping City forms the study area for our research and is the main location for economic development within the development zone. The gross domestic product (GDP) of Nanping City increased from 62.2 billion yuan in 2009 to 133.9 billion yuan in 2015, with an average annual growth of nearly 12 billion yuan due to the official establishment of the development zone in 2009. The rapid economic development and the acceleration of urbanization in Nanping City will inevitably affect the value of ecosystem services. Therefore, due to the high rate of urbanization, Nanping City was selected as the study area. The spatial differentiation characteristics between urbanization and the value of ecosystem services were analyzed using spatial measurement methods, and their relationships were investigated by applying the bivariate spatial autocorrelation method. Spatial measurement methods refers to a series of methods for studying various characteristics caused by space in the statistical analysis of regional scientific models [18-20]. This methods were used in this study to analyze population urbanization, spatial urbanization, economic urbanization, living urbanization and the spatial differentiation characteristics of ecosystem service value in the study area. The purpose of this study is to reveal the impact of the development of urbanization in Nanping City on the spatial heterogeneity of the value of ecosystem services, and to provide a reference for decision-making regarding regional urbanization construction and ecological environment management.

\section{Material and Methods}

\section{Study Area}

Nanping City $\left(26^{\circ} 15^{\prime}-28^{\circ} 19^{\prime} \mathrm{N}, \quad 117^{\circ} 00^{\prime}-119^{\circ} 17^{\prime} \mathrm{E}\right)$ covers an area of $26,300 \mathrm{~km}^{2}$, and it is the largest municipal area in Fujian Province (Fig. 1). The forest cover in Nanping is $74.75 \%$ and it has a subtropical monsoon climate, with the local mountainous area having a subtropical mountain climate. At the end of 2015, Nanping City had a registered population of 3.2 million of which 2.64 million were permanent urban residents), and the level of urbanization was $54 \%$.The city consists of two municipal districts, three county-level cities, and five counties, of which Jian'ou, Wuyishan, and Shunchang are called "Chinese Bamboo Towns", Jian'ou, Jianyang, and Zhenghe are known as "Chinese Castanea henryi Towns", and Shunchang is the only "Chinese Fir Town". Forestry plays an important role in the economy and is the main source of income for hill farmers. Nanping possesses five nature reserves at or above province level, covering an area of $720 \mathrm{~km}^{2}$. There are 1283 small nature reserves covering an area of $846.7 \mathrm{~km}^{2}$. Wuyishan City is one of the only four "World Natural and Cultural Heritage Sites" in China, and is not only a national scenic area 


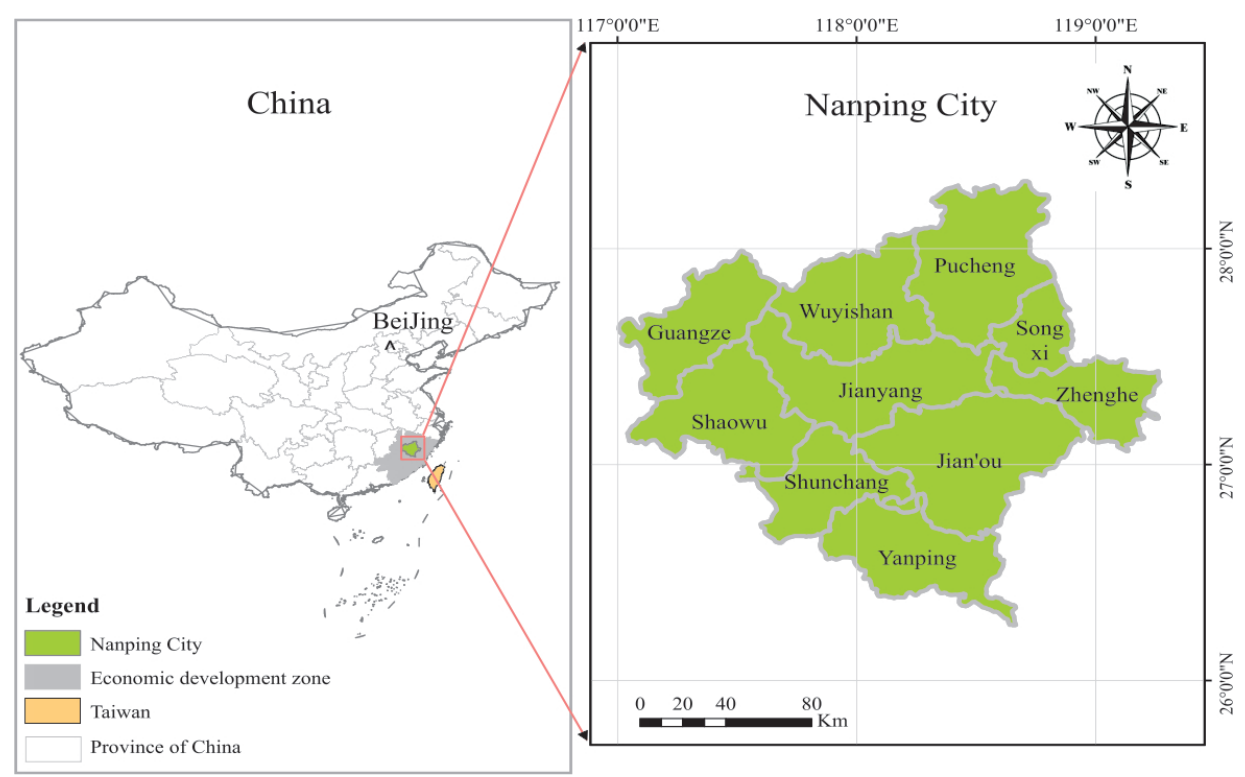

Fig. 1. Map of the study area.

and a nature reserve, but also falls within a tourism economic development zone in Fujian province.

\section{Data Source and Preprocessing}

Data regarding urbanization levels was taken from the 2015 statistical yearbook of Nanping City. The remote sensing image from Landsat-7 +ETM of Nanping City in 2015 was downloaded from the Geospatial Data Cloud Platform of the Chinese Academy of Sciences. The downloaded remote sensing image was preprocessed, including radiation correction, geometric correction, and mosaic and clipping of the image. The color synthesis of the study area was carried out using the 5, 4, and 3 bands, and the land use classification was performed using the object-oriented method. In this study, six land use types were used based on the natural conditions and land use characteristics of the study area, namely forest land, cultivated land, grassland, water, unused land, and construction land. It can be seen from the accuracy evaluation results that the Kappa coefficient is 0.9 , which lies between 0.8 and 1 , and indicates that the classification system has a high precision and can be used for further analyses.

\section{Determination of Urbanization Indicators}

Urbanization is mainly affected by indicators such as population growth (population urbanization), expansion of construction land (spatial urbanization), economic development (economic urbanization), and improvement in quality of life (living urbanization) [21]. Population urbanization, spatial urbanization, economic urbanization and living urbanization were respectively represented using the proportion of permanent residents, the proportion of construction land, the proportion of non-agricultural industries and disposable income of urban households.

Table 1. Equivalent value per unit area of terrestrial ecosystem services in China.

\begin{tabular}{|c|c|c|c|c|c|c|}
\hline Evaluation indicators & Forest land & Grassland & $\begin{array}{c}\text { Cultivated } \\
\text { land }\end{array}$ & Wetland & Water & Barren land \\
\hline Gas regulation & 3.50 & 0.80 & 0.50 & 1.80 & 0.00 & 0.00 \\
\hline Climate regulation & 2.70 & 0.90 & 0.89 & 17.10 & 0.46 & 0.00 \\
\hline Water conservation & 3.20 & 0.80 & 0.60 & 15.50 & 20.38 & 0.03 \\
\hline Soil formation and protection & 3.90 & 1.95 & 1.46 & 1.71 & 0.01 & 0.02 \\
\hline Waste treatment & 1.31 & 1.31 & 1.64 & 18.18 & 18.18 & 0.01 \\
\hline Biodiversity conservation & 3.26 & 1.09 & 0.71 & 2.50 & 2.49 & 0.34 \\
\hline Food production & 0.10 & 0.30 & 1.00 & 0.30 & 0.10 & 0.01 \\
\hline Supply of raw materials & 2.60 & 0.05 & 0.10 & 0.07 & 0.01 & 0.00 \\
\hline Entertainment and culture & 1.28 & 0.04 & 0.01 & 5.55 & 4.34 & 0.01 \\
\hline
\end{tabular}


Table 2. Equivalent value per unit area of terrestrial ecosystem services in Nanping City.

\begin{tabular}{|c|c|c|c|c|c|c|}
\hline Evaluation indicators & Forest land & Grassland & Cultivated land & Water & Unused land & Construction land \\
\hline Gas regulation & 3.50 & 0.80 & 0.50 & 0.00 & 0.00 & NA \\
\hline Climate regulation & 2.70 & 0.90 & 0.89 & 0.46 & 0.00 & NA \\
\hline Water conservation & 3.20 & 0.80 & 0.60 & 20.38 & 0.03 & NA \\
\hline Soil formation and protection & 3.90 & 1.31 & 1.46 & 0.01 & 0.02 & NA \\
\hline Waste treatment & 1.31 & 1.95 & 1.64 & 18.18 & 0.01 & NA \\
\hline Biodiversity conservation & 3.26 & 1.09 & 0.71 & 2.49 & 0.34 & NA \\
\hline Food production & 0.10 & 0.30 & 1.00 & 0.10 & 0.01 & NA \\
\hline Supply of raw materials & 2.60 & 0.05 & 0.10 & 0.01 & 0.00 & NA \\
\hline Entertainment and culture & 1.28 & 0.04 & 0.01 & 4.34 & 0.01 & NA \\
\hline
\end{tabular}

Population urbanization refers to the process of transforming rural population into urban population, and agricultural population into non-agricultural population. The proportion of permanent residents is the ratio of the population of various districts (counties) to the total population of Nanping City. The proportion of construction land refers to the ratio of the area of construction land in various districts (counties) to the total area of those districts (counties). The proportion of non-agricultural industries refers to the ratio of the sum of GDP of secondary and tertiary industries to the total GDP [22-24]. Disposable income of urban households refers to income that urban residents can freely control. It means the remaining income deducting the taxes and fees paid to the state from the total income of the resident family. These taxes and fees include medical insurance, old-age insurance, and unemployment insurance.

\section{Estimation of the Value of Ecosystem Services}

A table of the equivalent value per unit area of terrestrial ecosystem services in China (Table 1) was used in combination with the actual needs of this study and slightly modified to create a table of the equivalent value per unit area value of ecosystem services in Nanping City (Table 2). The value of ecosystem services for construction land was not calculated in this study. The main reason for this is that ecosystem services have a positive effect on humans, while construction land, such as rural residential land, urban industrial land, and mining land, causes great damage to the natural ecological environment due to the continuous discharge of sewage, waste gas, and solid waste into the environment. Therefore, it mainly has a negative effect on the ecological environment.

The average grain yield of Nanping City in 2015 $\left(5700 \mathrm{~kg} / \mathrm{hm}^{2}\right)$ and the average grain price in China (5.24 yuan $/ \mathrm{kg}$ ) were obtained by consulting a statistical yearbook of Nanping and related materials of the same year. The value of ecosystem services per unit area of cultivated land in Nanping City $\left(E_{a}, 4266.85\right.$ yuan $\left./ \mathrm{hm}^{2}\right)$ was determined by using Equation 1,

$$
E_{a}=\frac{1}{7} \cdot T_{a} \cdot T_{b}
$$

Table 3. Value per unit area of ecosystem services related to different land use types () in Nanping City (Ten thousands yuan/hm²).

\begin{tabular}{|c|c|c|c|c|c|}
\hline Evaluation indicators & Forest land & Cultivated land & Grassland & Water & Unused land \\
\hline Gas regulation & 1.4933 & 0.2133 & 0.3413 & 0.0000 & 0.0000 \\
\hline Climate regulation & 1.1520 & 0.3797 & 0.3840 & 0.1962 & 0.0000 \\
\hline Water conservation & 1.3670 & 0.2560 & 0.3413 & 8.6953 & 0.0128 \\
\hline Soil formation and protection & 1.6640 & 0.6229 & 0.5589 & 0.0042 & 0.0085 \\
\hline Waste treatment & 0.5589 & 0.7012 & 0.8320 & 7.7567 & 0.0042 \\
\hline Biodiversity conservation & 1.3909 & 0.3029 & 0.4651 & 1.0624 & 0.1450 \\
\hline Food production & 0.0427 & 0.4267 & 0.1280 & 0.0427 & 0.0042 \\
\hline Supply of raw materials & 1.1093 & 0.0427 & 0.0213 & 0.0042 & 0.0000 \\
\hline Entertainment and culture & 0.5461 & 0.0042 & 0.0171 & 1.8517 & 0.0042 \\
\hline Total & 9.3243 & 2.9497 & 3.0891 & 19.6136 & 0.1791 \\
\hline
\end{tabular}


...where $E_{a}$ is the ecosystem service value per hectare of cultivated land in Nanping City (yuan $/ \mathrm{hm}^{2}$ ), $T_{a}$ represents the average grain yield $\left(\mathrm{kg} / \mathrm{hm}^{2}\right)$, and $T_{b}$ is the average grain price in China.

The unit area value of a specific ecosystem service $\left(C V_{i j}\right)$ can be obtained by using Equation 2 and Table 2. Equation2 is as follows:

$$
C V_{i j}=E_{a} \cdot f_{i j}
$$

...where $C V_{i j}$ is a specific ecosystem service unit area value (yuan $/ \mathrm{hm}^{2}$.year) and $f_{i j}$ is the equivalence factor of a specific ecosystem service related to a specific land use type.

Using Table 3 and formula 3, the total value of ecosystem services in Nanping City can be obtained. Formula 3 is as follows:

$$
E S V=\sum_{i=1}^{n} \sum_{j}^{m} A_{i} \times C V_{i j}
$$

...where ESV is the total value of ecosystem services (yuan) and $\mathrm{A}_{i}$ denotes the area of a specific land use type.

\section{Analysis of Bivariate Spatial Autocorrelation}

Spatial autocorrelation analysis includes global spatial autocorrelation and local spatial autocorrelation [25]. Global spatial autocorrelation is used to describe the average degree of association, spatial distribution patterns, and significance of all objects in the study area. The local spatial autocorrelation statistical variables can identify spatial association patterns that may exist in different spatial locations, thus discovering the instability of local space. It can more accurately grasp the aggregation and differentiation characteristics of local spatial features, providing a basis for the sake of classification and decision-making [26-27]. In this study, global spatial autocorrelation and local spatial autocorrelation were described by using Moran's I and Local Moran's I, respectively. Among them, the Moran's I can be calculated using Equation 4,

$$
I=\frac{\sum_{i=1}^{n} \sum_{j \neq i}^{n} W_{i j} \cdot\left(Y_{i}-\bar{Y}\right) \cdot\left(Y_{j}-\bar{Y}\right)}{S^{2} \cdot \sum_{i=1}^{n} \sum_{j \neq i}^{n} W_{i j}}
$$

For a single spatial unit $i$, the Local Moran's I can be calculated using Equation 5,

$$
I_{i}=\frac{Y_{i}-\bar{Y}}{S_{i}^{2}} \cdot \sum_{i=1, j \neq i}^{n} w_{i j} \cdot\left(Y_{i}-\bar{Y}\right)
$$

$\ldots$ where $S^{2}=\frac{1}{n} \sum_{i=1}^{n}\left(Y_{i}-\bar{Y}\right), \bar{Y}=\frac{1}{n} \sum_{i=1}^{n} Y_{i}, Y_{i}, Y_{i}$ and $Y_{j}$ denote the attribute values of unit $i$ and unit $j$, respectively, $n$ is the number of spatial units, and $W_{i j}$ is a weight matrix based on spatial adjacency relationships.

Related scholars [28-30] further expanded the bivariate global autocorrelation and local autocorrelation based on the Moran's I to characterize the spatial correlation between multiple variables. This provides a feasible method to reveal the correlation of the spatial distribution of different elements. The formula is as follows:

$$
I_{l m}^{p}=Z_{l}^{p} \cdot \sum_{q=1}^{n} W_{p q} \cdot Z_{m}^{q}
$$

...where $Z_{l}^{p}=\frac{X_{l}^{p}-\overline{X_{l}}}{\sigma_{l}}, Z_{m}^{q}=\frac{X_{m}^{q}-\overline{X_{m}}}{\sigma_{m}}, X_{l}^{p}$ is the value of attribute $l$ of spatial units $p, X_{w}^{p}$ is the value of attribute $m$ of spatial units $q, \overline{X_{l}}$ and $\bar{X}_{m}$ are the average value of attribute $l$ and $m$, respectively, and $\sigma_{l}$ and $\sigma_{m}$ are the variance of the attribute of $l$ and $m$, respectively.

\section{Results and Discussion}

\section{Spatial Distribution of Urbanization Indicators}

To investigate the spatial distribution of urbanization indicators, each unit value was divided by the natural discontinuous point classification (Jenks). It can be seen from Fig. 2, in general, that the overall level of urbanization in Nanping City was highest in Yanping. At the same time, compared to other regions, the level of urbanization in the central region was slightly higher than in the surrounding regions.

In terms of population urbanization (Fig. 2a), the highest percentage was found in the south, with Yanping and Jian'ou reaching $17.88 \%$ and $17.08 \%$ respectively, whereas the urbanization rate of the population in Guangze and Songxi - located in the western and eastern parts of the study area, respectively - was less than $5 \%$. This indicates that the population concentration level in these regions was low.

Regarding spatial urbanization, Fig. 2b) shows an increase from west to east. A contiguous low-level spatial urbanization pattern was found in Guangze, Shaowu, and Shunchang (in the western part of the study area), with a spatial urbanization value of less than 3\%. Songxi and Zhenghe in the eastern part of the study area presented a higher percentage of spatial urbanization, namely $14.94 \%$ in Songxi and $14.02 \%$ in Zhenghe.

Concerning economic urbanization (Fig. 2c), Yanping had the highest level of economic urbanization at $87.35 \%$, while Guangze had the lowest level at $52.98 \%$. In contrast, the economic urbanization levels of other regions gradually increased from east to west.

In terms of living urbanization (Fig. 2d), the urbanization level of Shaowu and Yanping was the 

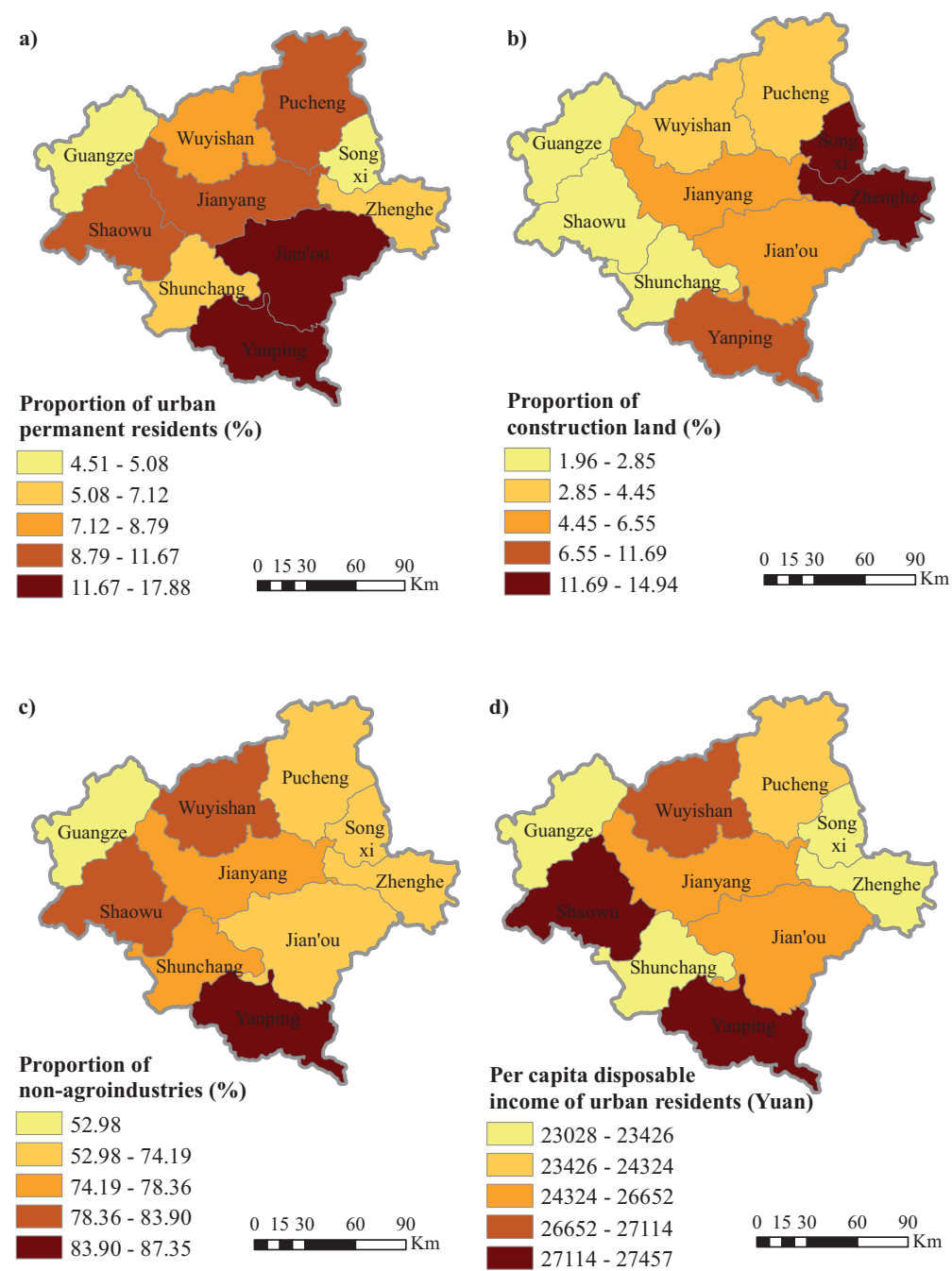

Fig. 2. Spatial distribution of urbanization in Nanping city.

a) Population urbanization, b) Spatial urbanization, c) Economic urbanization, d) Living urbanization.

highest. The disposable income of urban households in Shaowu and Yanping was 27,457 yuan and 27,395 yuan, respectively. However, the living urbanization level of Songxi, Zhenghe, Guangze, and Shunchang was lower than that of Shaowu and Yanping, with the disposable income of urban households of urban residents being 23,028 yuan in Songxi. This was nearly $20 \%$ lower than that of Shaowu which had the highest living urbanization level.

In summary, the population, economic, and living urbanization levels were higher in Yanping than in other regions. This is mainly because Yanping is the seat of the municipal government. Urban development tends to spread around the government as the center. Songxi, located in the northeastern region of Nanping, had the highest spatial urbanization percentage, but had the lowest population urbanization and living urbanization levels. Guangze, located in the northwestern region of the study area, had the lowest levels of all four urbanization indicators which points out that it had the lowest level of urbanization.

\section{Spatial Distribution of the Value of Ecosystem Services}

Fig. 3a) shows that the value of ecosystem services was the highest in Jian'ou with 27.136 billion yuan and the lowest in Songxi with 7.343 billion yuan, nearly four times lower than in Jian'ou. Puyang in the north and Jianyang in the central region had a high value of ecosystem services, while the value of ecosystem services in Wuyishan, Yanping, and Shaowu in the north, south, and west of the study area, respectively, were at a medium level. Furthermore, Guangze and Shunchang in the northwestern and southwestern regions of the study area, respectively, represented a low level in the value of ecosystem services, while, in contrast, Songxi and Zhenghe in the east of the study area reflected the lowest value of ecosystem services.

In this study, the per kilometer value of ecosystem services were calculated in the study area to eliminate the area dependence in the value of ecosystem services (Fig. 3b). The spatial distribution pattern of the per kilometer value of ecosystem services was high in the 

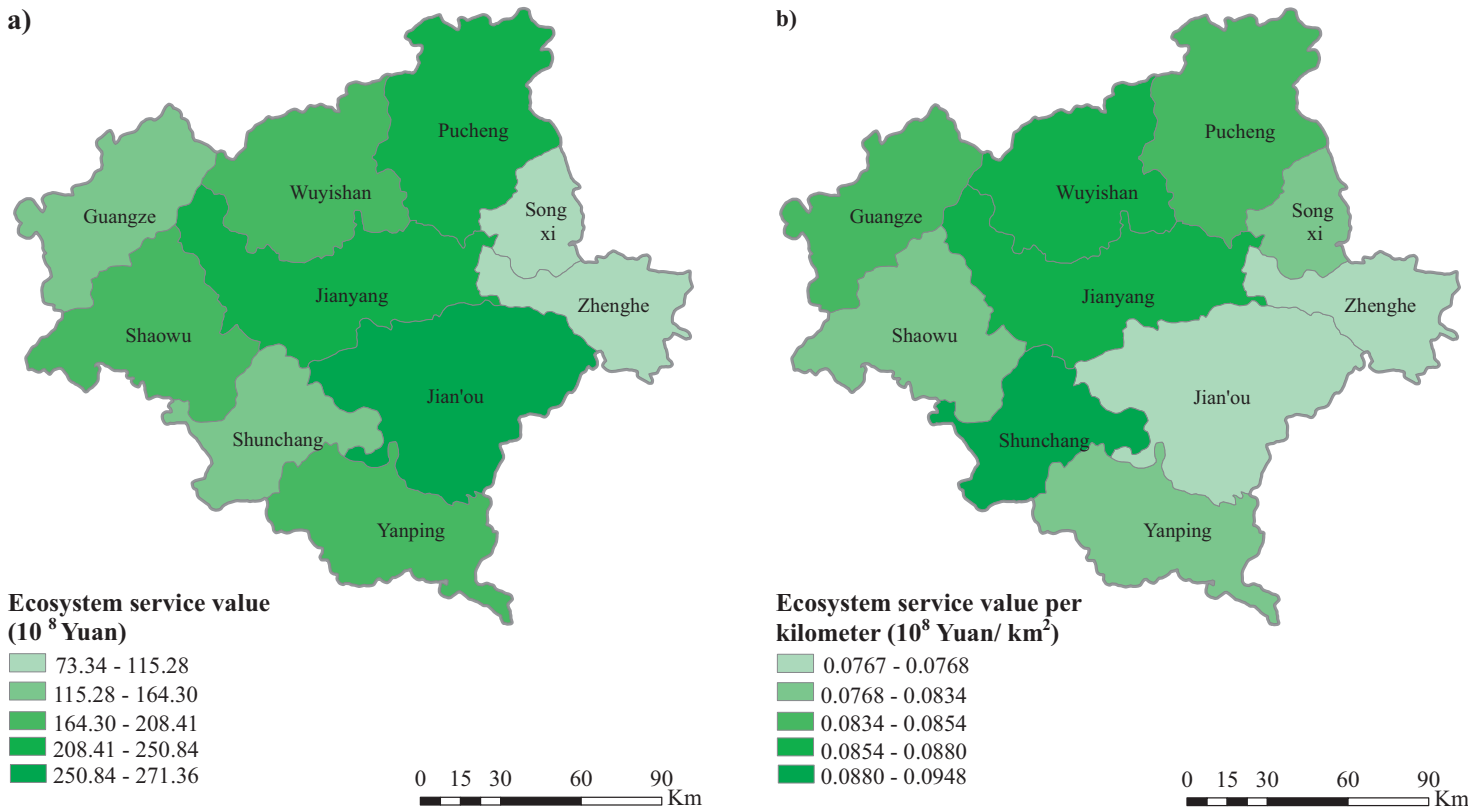

Fig. 3. Spatial distribution of the value of ecosystem services in Nanping city. a) Ecosystem service value, b) Ecosystem service value per kilometer.

central and northern parts of Nanping City and low in the southeastern parts. Specifically, the per kilometer value of ecosystem services was the highest in Shunchang at $0.0948 \times 10^{8}$ yuan, while the value was the lowest in Jian'ou with $0.0767 \times 10^{8}$ yuan. In contrast, the value was higher in Jianyang and Wuyishan, located in the central and northern parts of the study, respectively. The main reasons for these values are that Shunchang is the only "Chinese Fir Town", and Jianyang is known as the "Forest World", one of the key forest areas in southern China. Furthermore, Wuyishan is a world heritage site in terms of nature and culture, and has preserved the largest, most intact, and characteristic sub-tropical native forest ecosystem compared to other forest ecosystems at the same latitude in the world. Compared to other regions, Shunchuang, Jianyang, and Wuyishan had higher forest coverage and more favorable ecological resources, and thus had a higher per kilometer value of ecosystem services.

\section{Bivariate Global Spatial Autocorrelation}

The global spatial autocorrelation between different urbanization levels and the value of ecosystem services was calculated using the spatial analysis tool GeoDa in order to establish the spatial weight matrix. As can be seen from Table 4, the bivariate Moran's I of the four urbanization indicators and the total value of ecosystem services was less than zero. Among them, the bivariate spatial correlation between population urbanization and the total value of ecosystem services was significant, indicating a significant negative spatial correlation between them. The bivariate spatial correlation Moran's I value was -0.1385 , which suggests that the gradual movement of the agricultural population to urbanized areas was the main cause of the decline in the value of ecosystem services. However, the bivariate Moran's I index of the economic, spatial, and living urbanization levels and the total value of ecosystem services did not pass the significance test, which indicates that there was no significant spatial correlation between them.

From the perspective of the bivariate spatial autocorrelation considering both urbanization and the individual event service function, there was a certain negative correlation in the population urbanization level and the individual event service function. Among them, the strongest correlation was found between population urbanization and food production, with a Moran's I index of -0.1793 . This is mainly because the shortage of rural surplus labor force is one of the reasons for the reduction in food production capacity after the agricultural population converting to cities during the process of urbanization. Therefore, in light of the urbanization process, government should be more vigilant about the food shortages caused by rapid population growth in the city. In addition, there was a significant negative correlation between population urbanization and gas regulation as well as soil formation and protection, mainly due to population accumulation leading to the degradation of ecosystem functions.

Additionally, there was a positive correlation between economic urbanization and waste treatment, with a Moran's I index of 0.1163 . This indicates that the process of economic development in the study area had not caused great harm to the environment, with a focus on the centralized management and comprehensive utilization of pollutants and wastes. In contrast, there was no obvious correlation between spatial urbanization 


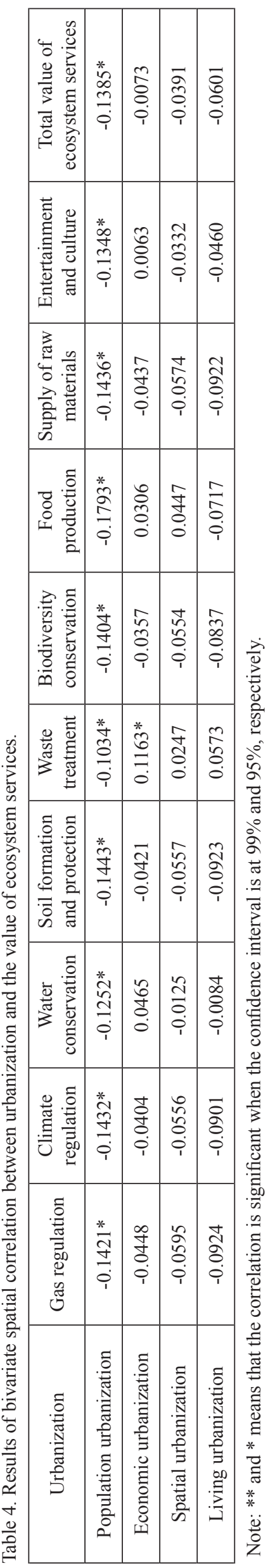

and living urbanization with the individual event service function.

\section{Bivariate Local Spatial Autocorrelation}

The bivariate local spatial autocorrelation can be used to characterize the relationship between the regional urbanization level and the mean value of its neighborhood ecosystem services. The relationships include positive correlation, negative correlation, and no significant correlation. Specifically, a positive correlation described a high urbanization level with a high ecosystem services value (High-High) and a low urbanization level with a low ecosystem services value (Low-Low), while a negative correlation included a low urbanization level together with a high ecosystem services value (Low-High) and a high urbanization level with a low ecosystem services value (High-Low). The bivariate local index of spatial association (LISA) aggregate map was plotted on the basis of the $\mathrm{Z}$ test $(p=0.05)$ (Fig. 4). From Fig. 4a, it can be seen that Guangze showed a Low-High correlation in terms of population urbanization and the value of ecosystem services, while Jian'ou presented a High-Low correlation, and there was no significant correlation in other regions between population urbanization and the value of ecosystem services. Only Shaowu showed a High-Low correlation between spatial urbanization and the value of ecosystem services, characterized by an obvious high spatial urbanization-low ecosystem services value (See Fig. 4b for details). Only one spatial distribution pattern between economic urbanization and ecosystem services value was similar to that of population urbanization (refer to Fig. 4c). That is to say, Shunchang presented a Low-High correlation in terms of economic urbanization and the value of ecosystem services. In contrast, the regions that showed a low living urbanization-high ecosystem services correlation were Guangze and Shunchang (Fig. 4d).

In all, Jian'ou and Shaowu were characterized by a high urbanization level-low ecosystem services value, while Guangze and Shunchang were characterized by a low urbanization level-high ecosystem services value. Whereas, Jian'ou and Shaowu should fully utilize the advantage of being "Chinese Bamboo Towns", strengthen the protection and ecological wellbeing of their forests, improve the value of ecosystem services and functions, control the scale and the speed of the expansion of urban construction, and minimize the destruction of ecosystems whilst promoting urbanization, Guangze and Shunchang should steadily promote urbanization and gradually move towards the simultaneous development of urbanization as well as the ecosystem on the basis of maintaining the existing ecological advantages. In conclusion, in view of the targets of the green hinterland of the economic development zone on the west side of the strait, the effective way to realize the coordinated development between urbanization and the protection of ecosystems 

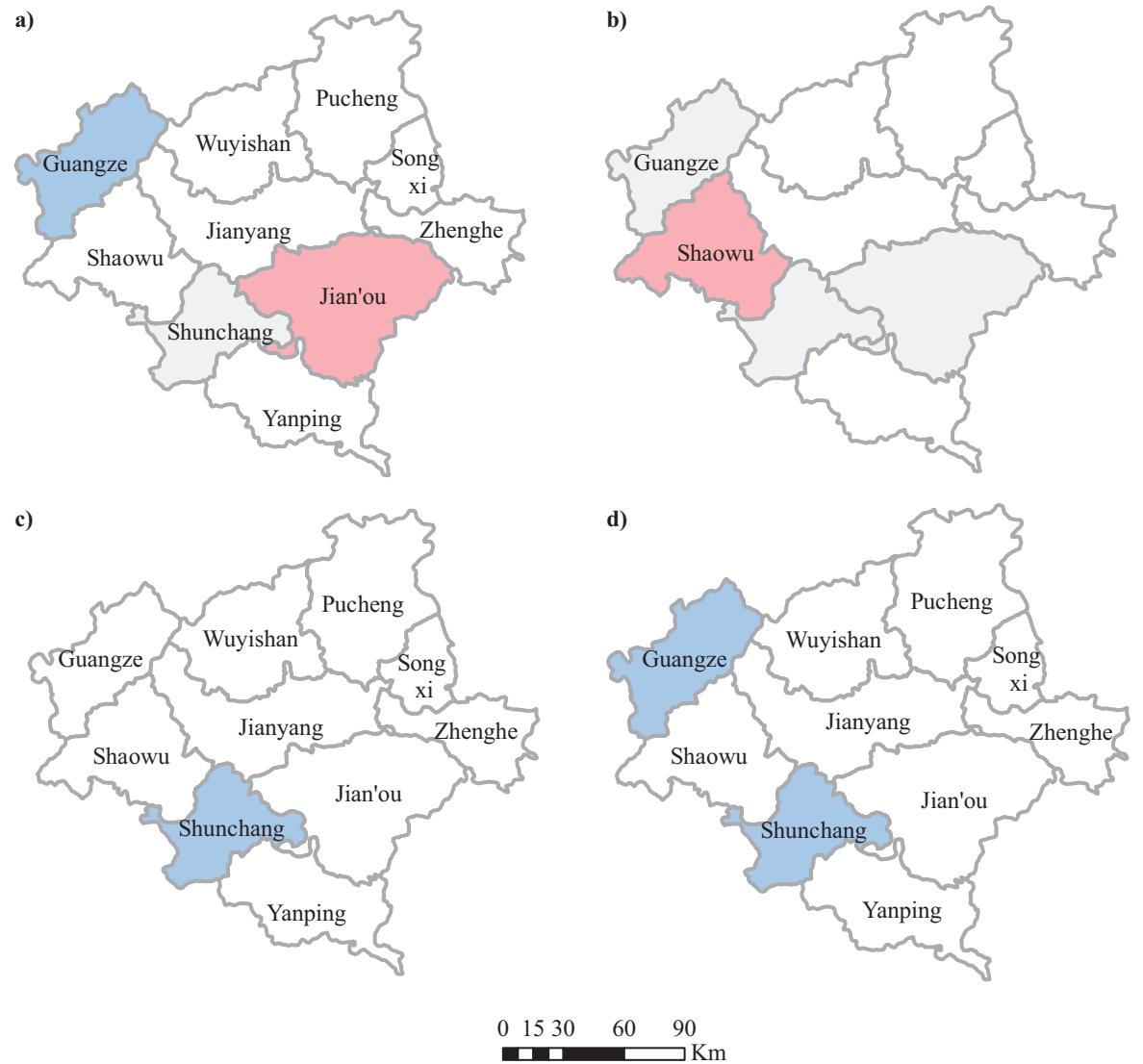

\begin{tabular}{|l|l|l|l|l}
$\square$ Not Significant \\
$\square$
\end{tabular}

Fig. 4. Bivariate LISA cluster map of urbanization with ESV. a) Population urbanization with ESV, b) Spatial urbanization with ESV, c) Economic urbanization with ESV, (d) Living urbanization with ESV.

is to take various actions, amongst others the strengthening of the protection of natural ecosystems, the improvement of the living environment, the development of a circular economy, and the prevention and control of pollutants.

Based on previous studies [22-24], levels of population, spatial, economic, and living urbanization were described by selecting the disposable income of urban households and the proportion of permanent residents, construction land, and non-agricultural industries, which were easy to calculate and had the strongest influence on urbanization. However, there were some limitations to measuring the level of urbanization with the above single indicator. With the continuous improvement of various statistics related to Nanping city, the examination of the different aspects of urban development through the use of various indicators and a comprehensive analysis of certain characteristics of the city should be urgently addressed in the future.

The equivalent value per unit area of terrestrial ecosystem services in China was corrected in this paper. It provides a feasible way to explore the correlation between ecosystem service value and urbanization in Nanping City. However, as far as the estimation of ecosystem service value is concerned, it still has some shortcomings. For example, different correction methods and parameters in the same research area will cause differences in evaluation results, and local differences are also difficult to measure with unified parameters. Therefore, it should be one of study directions for further exploration to conduct systematic research on the spatial transformation and scale effect of ecosystem service value adopting a more accurate assessment model in order that improving the accuracy of ecosystem service value assessment.

\section{Conclusions}

Based on the connotation of urban development, the urbanization development pattern of Nanping City was investigated in terms of the levels of population, spatial, economic, and living urbanization. The bivariate spatial autocorrelation method was used to analyze the impact of urbanization on the value of ecosystem services and the following main conclusions were reached:

The highest level of urbanization in Nanping City was broadly located in Yanping, with the level of 
urbanization in the central regions being higher than in the other regions. Concerning the per kilometer value of ecosystem services, Shunchang in the southwest of the study area had the highest value, while Jian'ou reflected the lowest value. Furthermore, the per kilometer value of ecosystem services in Jianyang and Wuyishan, located in the central and northern parts of the study area, respectively, had higher levels of the per kilometer value of ecosystem services than the other regions.

There was a significant negative spatial correlation between the level of population urbanization and the total value of ecosystem services, while there was no significant spatial correlation between the levels of economic urbanization, spatial urbanization, and living urbanization and the total value of ecosystem services. Regarding the individual event service value and all levels of urbanization, there was a certain negative correlation between the level of population urbanization and the individual event service value, with the correlation between food production being the strongest. At the same time, there was a positive correlation regarding both economic urbanization and waste treatment, while there was no obvious correlation between spatial urbanization and living urbanization and the individual event service value.

From the perspective of the bivariate LISA aggregate map, Jian'ou and Shaowu showed a high urbanization level-low ecosystem services value, and a low urbanization level-high ecosystem services value appeared in Guangze and Shunchang.

\section{Acknowledgments}

We are so grateful for the financial support provided by the Specialized Research Fund of the Doctoral Program of Shaoyang University (Fund No. 17ZX03), the Education Department of Hunan Province (No.19C1670), and the National Science and Technology Major Project of the Ministry of Science and Technology of China (Fund No. 2017YFD0601304) . The authors wish to thank the referees for providing helpful suggestions in improving this manuscript.

\section{Conflict of Interest}

No conflict of interest exits in the submission of this manuscript, and manuscript is approved by all authors for publication.

\section{References}

1. HOU Q., ZHU W., LIU X., ZHANG X. Urban ecosystem health assessment inside Xi'an by multivariate data. J Environ Prot Ecol, 19 (3), 1016, 2018.

2. COSTANZA J.K., TERANDO A.J., MCKERROW A.J., COLLAZO J.A. Modeling climate change, urbanization, and fire effects on pinus palustris ecosystems of the Southeastern U.S. J Environ Manage, 151, 186, 2015.

3. ALI H.S., LAW S.H., ZANNAH T.I. Dynamic impact of urbanization, economic growth, energy consumption, and trade openness on $\mathrm{Co}_{2}$ emissions in Nigeria. Environ Sci \& Pollut, 23 (12), 12435, 2016

4. WU T., PERRINGS C., KINZIG A., COLLINS J.P., MINTEER B.A., DASZAK P. Economic growth, urbanization, globalization, and the risks of emerging infectious diseases in China: A review. Ambio, 46 (1), 18, 2017.

5. LIN Y.C., LI Y.L., MA Z.L. Exploring the interactive development between population urbanization and land urbanization: evidence from Chongqing, China (19982016). Sustainability, 10 (6), 1741, 2018.

6. ANDRÉS M.D., BARRAGÁN J.M., SANABRIA J.G. Relationships between coastal urbanization and ecosystems in Spain. Cities, 68, 8, 2017.

7. HOU L., WU F.Q., XIE X.L. The spatial characteristics and relationships between landscape pattern and ecosystem service value along an urban-rural gradient in Xi'an city, China. Ecol Indic, 108, 1, 2020.

8. COSTANZA R., GROOT R.D., SUTTON P., PLOEG S.V.D., ANDERSON S.J., KUBISZEWSKI I., FARBER S., TURNER R.K. Changes in the global value of ecosystem services. Global Environ Chang, 26 (1), 152, 2014.

9. ZHANG Y., LIU Y., ZHANG Y., LIU Y., ZHANG G., CHEN Y. On the spatial relationship between ecosystem services and urbanization: A Case Study in Wuhan, China. Sci Total Environ, 637-638, 780, 2018.

10. HE J., WANG S., LIU Y., MA H., LIU Q. Examining the relationship between urbanization and the ecoenvironment using a coupling analysis: case study of Shanghai, China. Ecol Indic, 77, 185, 2017.

11. CIVEIRA G., LADO L.M., VAZQUEZ E.V., GONZALEZ A.P. Ecosystem services and economic assessment of land uses in urban and Periurban Areas. Environ Manage, 65 (3), 355, 2010.

12. HOMER C., DEWITZ J., JIN S., XIAN G., COSTELLO C., DANIELSON P., GASS L., FUNK M., WICKHAM J., STEHMAN S., AUCH R., RIITTERS K. Conterminous United States land cover change patterns 2001-2016 from the 2016 national land cover database. ISPRS J Photogramm, 162, 184, 2020.

13. ZHAO Q.J., WEN Z.M., CHEN S.L., DING S., ZHANG M.X. Quantifying land use/land cover and landscape pattern changes and impacts on ecosystem services. Int $\mathrm{J}$ Environ Res Public Health, 17, 126, 2020.

14. ABDULLAH A.Y.M., MASRUR A., ADNAN M.S.G., BAKY M.A.A., HASSAN Q.K., DEWAN A. Spatiotemporal patterns of land use/land cover change in the heterogeneous coastal region of Bangladesh between 1990 and 2017. Remote Sens, 11, 790, 2019.

15. LIU Y.B., HOU X.W., SONG B.Y., WANG C. Assessing and predicting changes in ecosystem service values based on land use/cover change in the Bohai Rim coastal zone. Ecol Indic, 111, 1, 2020.

16. GONZÁlEZ-GARCÍA A., PALOMOA I., JOSÉ A., GONZÁLEZ J.A., LÓPEZ C.A., MONTES C. Quantifying spatial supply-demand mismatches in ecosystem services provides insights for land-use planning. Land Use Policy, 94, $1,2020$.

17. DEWAN A.M., YAMAGUCHI Y. Land use and land cover change in Greater Dhaka, Bangladesh: Using remote sensing to promote sustainable urbanization. Appl Geogr, 29, 390, 2009. 
18. ANSELIN L. Model validation in spatial econometrics: A review and evaluation of alternative approaches. Int Regional Sci Rev, 11 (3), 279, 1988.

19. XU Y., LI A. The relationship between innovative human capital and interprovincial economic growth based on panel data model and spatial econometrics. J Comput Appl Math, 365, 1, 2019.

20. ANTONAKAKIS N. Recent developments in spatial econometrics. J Geogr Syst, 22 (1), 3, 2020.

21. LEE S.D., MILLER-RUSHING A.J. Degradation, urbanization, and restoration: A review of the challenges and future of conservation on the Korean Peninsula. Biol Conserv, 176 (176), 262, 2014.

22. FANG C.L., CUI X.G., LI G.D. Modeling regional sustainable development scenarios using the urbanization and eco-environment Coupler: case study of Beijing Tianjin-Hebei urban agglomeration, China. Sci Total Environ, 689, 820, 2019.

23. SHAN Z., HUANG Y. An analysis of the concept, goals, contents, planning strategies and misunderstandings of new urbanization. Urban Planning Forum, 288 (2), 16, 2013.
24. LIU H.B., LIU Y.F., WANG H.N., YANG J.Y., ZHOU X. Research on the coordinated development of greenization and urbanization based on system dynamics and data envelopment analysis - a case study of Tianjin. J Clean Pro, 214, 195, 2019.

25. YAO X., ZENG J., LI W. Spatial correlation characteristics of urbanization and land ecosystem service value in Wuhan Urban Agglomeration. Transactions of the Chinese Society of Agricultural Engineering, 31 (9), 249, 2015.

26. XIE G., LU C., LENG Y., ZHENG D., LI S. Ecological assets valuation of the Tibetan Plateau. Journal of Natural Resources, 18 (2), 189, 2003.

27. DORMANN C. Effects of incorporating spatial autocorrelation into the analysis of species distribution data. Global Ecol Biogeogr, 16 (2), 129, 2007.

28. SOKAL R.R., ODEN N.L., THOMSON B.A. Local spatial autocorrelation in biological variables. Biol J Linn Soc, 65 (1), 41, 1998.

29. ANSELIN L. Local indicators of spatial association - Lisa. Geogr Anal, 27 (2), 93, 1995.

30. WARTENBERG D. Multivariate Spatial Correlation: A method for exploratory geographical analysis. Geogr Anal, 17 (4), 263, 2010. 
